\title{
The Quantification of Monacolin K in Some Red Yeast Rice from Fujian Province and the Comparison of the Other Product
}

\author{
Hong-Nan HuAng, ${ }^{*, a, b}$ Yong-You HuA, ${ }^{b}$ Guo-Rong BAO, ${ }^{c}$ and Lian-Hui XIE ${ }^{a}$ \\ ${ }^{a}$ Institute of Plant Virology, Fujian Agriculture and Forestry University; Jinshan Fuzhou 350002, China: ${ }^{b}$ Department of \\ Physical and Chemical Analysis, Fujian Center for Disease Control and Prevention; Fuzhou, 350001, P. R. of China: and \\ ${ }^{c}$ Department of Pharmacy, Fujian College of Traditional Chinese Medicine; 282 Wusi road Fuzhou, 350003, P. R. of \\ China. Received August 24, 2005; accepted December 19, 2005
}

When used as a dietary supplement to achieve and maintain healthy cholesterol levels, Chinese red yeast rice has significant potential to reduce health care costs and contribute to public health by reducing heart disease risk in individuals with moderate elevations of circulating cholesterol levels. Red yeast rice is typical product in Mingbei area of Fujian province. Nine products from different area were measured, using high-performance chromatography (HPLC) with photodiode array detector (PDA) and tandem mass spectrometry (MS) and the results show that the contents of monacolin $K$ in these products were considerably different, and more than the other product.

Key words Red yeast rice; monacolin K; HPLC; photodiode array detector (PDA); MS

Red yeast rice, a traditional Chinese medicine and food known for centuries to improve blood circulation and maintain taste and color in fish and meat. Preparation of red yeast rice following ancient methods by fermenting the fungal strain Monascus purpureus went on moist and sterile rice. The medicinal properties of red yeast rice were described by pharmacologists of the Ming Dynasty (1368-1644) as cited by Ma et $a l .^{1-5)}$ It is concluded from the present evidence that Red yeast rice possess opportunities for development as new functional foods. ${ }^{6}$ ) Red yeast rice, or Hongqu in Chinese, is a typical product in Mingbei area of Fujian province, and a most well known product from Gutiang. ${ }^{7)}$ From ancient time to now, the method of production all over the countries including Taiwan, and Okinawa (Japan) has been originating from Gutiang and Pinnan counties of Fujian province. ${ }^{7)}$ The modern research has revealed that monacolin $\mathrm{K}$ has been proven to be a characteristic constituent of red yeast rice. ${ }^{8)} \mathrm{In}$ this report, monacolin $\mathrm{K}$ was identified in red yeast rice of Fujian Province, using high-performance liquid chromatography with photodiode array detector and tandem mass spectrometry. All of these materials including raw material powder and finished products available from the different areas of Fujian province were quantified and the contents of monacolin $\mathrm{K}$ were calculated with reference of monacolin $\mathrm{K}$ commercially available as the standard. ${ }^{9,10)}$

\footnotetext{
Experimental

Chemicals, Reagents and Materials Methanol, HPLC grade; de-ionized water was obtained with an in-house Milli-Q water system (Millipore Inc., U.S.A.). Monacolin K standard was ordered from Sigma (MO, U.S.A.), nine batches of red yeast rice powder were kindly provided by a health food company in Fuzhou, commercial product from the other province of China.

Equipment Liquid chromatography and mass spectrometry (LC/ PDA/MS) were carried out using a system equipped with Surveyor LC system (Thermo Finnigan, San Jose, U.S.A.) including quaternary pump, online degasser, column heater compart, autosampler and photodiode array detector, and a LCQ DECA XP MAX mass spectrometer (Thermo Finnigan, San Jose, U.S.A.) that consists of an ESI interface and an ion trap mass analyzer. The software for the control of the equipment, and the acquired and treatment of data is Xcalibur 1.3 workstation.

The chemical fingerprints were conducted on the HPLC system of Waters 2695 Alliance (Milford, MA, U.S.A.), consisting of quaternary pump solvent management system, an on-line degasser and an autosampler. The raw data were detected by 2996 photo-diode array detector (PDA), and acquired and
}

processed by Waters Empower chromatographic workstation loaded on a Compaq computer. Prior to each run, the HPLC-PDA system was allowed to warm up for nearly $30 \mathrm{~min}$ and the baseline was monitored until stable before the samples were injected.

Sample Preparation About $0.3 \mathrm{~g}$ red yeast rice powder, accurately weighed, was transferred to a $10-\mathrm{ml}$ tampon centrifuge tube. The preparations were extracted with $10 \mathrm{ml}$ of $75 \%$ methanol for $60 \mathrm{~min}$ on an ultrasonic bath and kept standing for $30 \mathrm{~min}$ and then subsequently centrifuged for $6 \mathrm{~min}$ at $6000 \mathrm{rpm}$. The final solution was filtered through a $0.45-\mu \mathrm{m}$ membrane before injection.

Preparation of the Standard Solution Calibration curves were obtained from purified monacolin $\mathrm{K}$. The purity of the standard was evaluated by HPLC-PDA ${ }^{11)}$ with detection at $238.6 \mathrm{~nm}$. By recording the peak index via photodiode array detection, the purity was found to be greater than $99 \%$. Monacolin K (10 mg, weighed accurately) was dissolved in mobile phase (75\% methanol) in a $50 \mathrm{ml}$ actinic volumetric flask. This solution was diluted with $75 \%$ methanol to obtain standard solutions for the calibration curve in a range of $0.2-20 \mathrm{ng}$ on column $(n=6)$, and a $20-\mu 1$ aliquot was injected. The amount of monacolin $\mathrm{K}$ was calculated using the calibration curve of monacolin $\mathrm{K}$.

Method Development The chemical profiling procedure conducted on the liquid chromatography with photodiode array detector were optimized by testing various system conditions. General reverse-phase $\mathrm{C}_{18}$ column was used, and several different elution systems were trialed. The resolution and symmetry factors of peak as monacolin $\mathrm{K}$ with theirs neighboring peak were dissatisfied by using methanol-water or acetonitrile-water system. The results suggested a system composed of $75 \%$ methanol as an ideal system for the separation of the sample solution. For the consideration of resolution, the column of Merck LiChroCART RP-18 $(250 \mathrm{~mm} \times 4.6 \mathrm{~mm}$ i.d. $5 \mu \mathrm{m})$ was used. The chromatography was performed using mobile phase of $75 \%$ methanol. The total analysis time was $30 \mathrm{~min}$, including column stabilization. The photo-diode array detector was set at $210-350 \mathrm{~nm}$ and the chromatogram detected at $238.6 \mathrm{~nm}$. The column temperature was set at $30^{\circ} \mathrm{C}$, and the injection volume was $10 \mu \mathrm{l}$.

The chromatographic condition of LC/PDA/MS was modified from the condition of LC/PDA ${ }^{12)}$ The separations by LC/PDA/MS were performed on an arrow-bore reversed-phase BioBasic-18 pioneer HPLC column $(2.1 \mathrm{~mm} \times 150 \mathrm{~mm}$ i.d., $5 \mu \mathrm{m}$, Thermo corporation) with a $75 \%$ methanol elution at a flow rate $300 \mu \mathrm{l}$ per minute. The elution from the HPLC was passed PDA and then directly introduced into the mass spectrometry using eletrospray ionization (ESI). All the analyses were performed using ESI interface with the following setting: positive ionization mode; the temperature of the capillary is $350^{\circ} \mathrm{C}$; spray voltage is $4.5 \mathrm{kv}$; capillary voltage is $22 \mathrm{~V}$; sheath gas (N2) flows at 30 A.U. The electrospray interface and mass spectrometric parameters were optimized to obtain maximum sensitive unit resolution. 

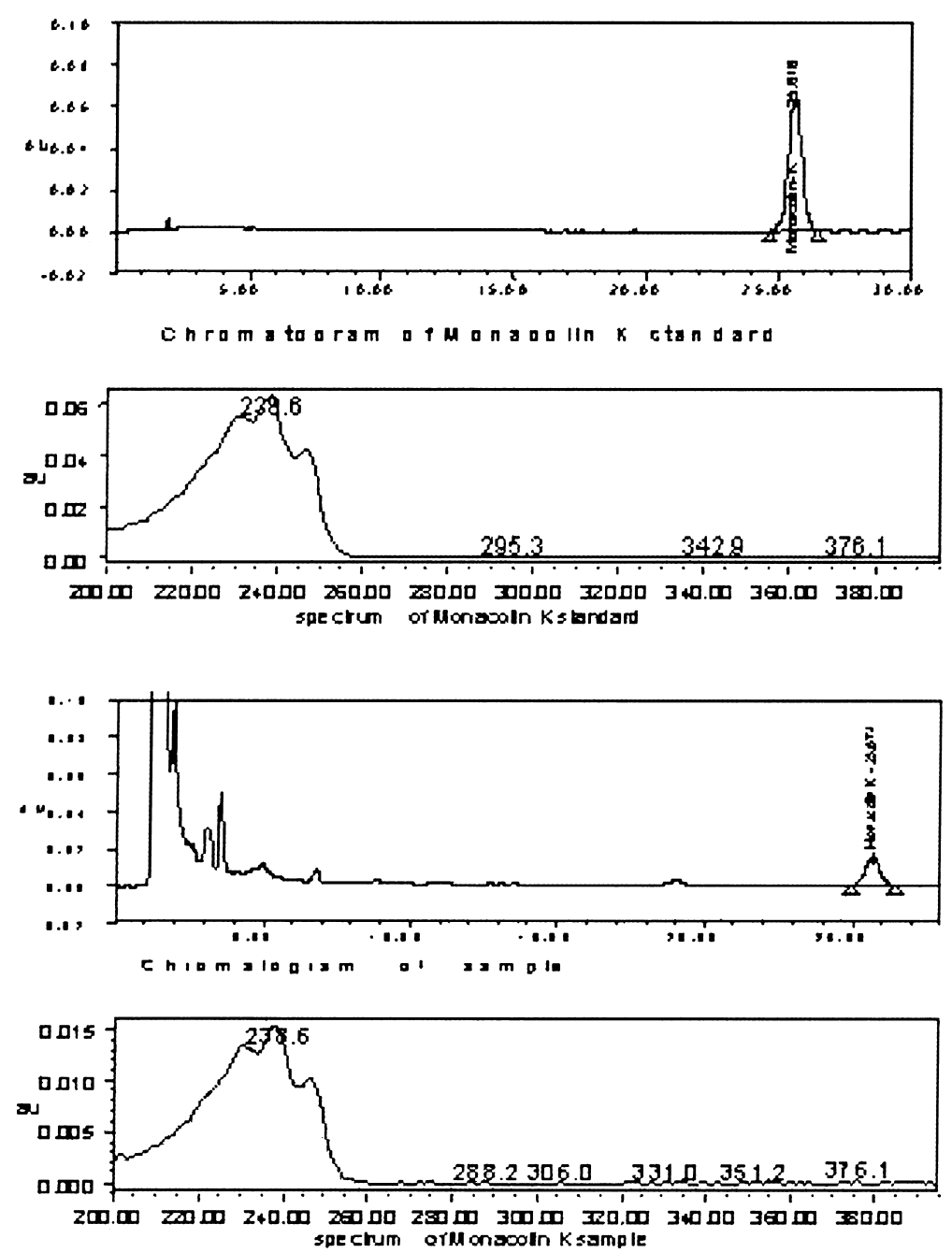

Fig. 1. The Chromatogram and Spectrum of Monacolin K Standard and Sample

\section{Results and Discussion}

Chromatographic Peak Identification. Identification by $\mathbf{L C} / \mathbf{P D A}$ Fermented red rice sample preparations were traced by HPLC/PDA, and chromatogram with on-line UV spectra. Results were shown in Fig. 1. The chromatogram was detected at wavelength of $238.6 \mathrm{~nm}$ and on-line UV spectra from 210 to $360 \mathrm{~nm}$. The peak of monacolin $\mathrm{K}$ in samples was identified by comparing to chromatogram, and the UV spectrum obtained with the monacolin $\mathrm{K}$ standard which showed a characteristic mountain-like spectrum with three maximum absorptions at $\left(\lambda_{\max }\right) 230,238.6$, and $246 \mathrm{~nm}$, respectively.

Identification by LC/PDA/MS The red yeast rice preparation was traced by hyphenated instrumentation of liquid chromatography with mass spectrometry. Figure 2 showed the mass spectrum of monacolin $\mathrm{K}$ and red yeast rice preparation of Fujian Province. The molecular ion of the predominant peak was $427(\mathrm{~m}+23), \mathrm{Na}(\mathrm{MW}=23)$ identified as<smiles>CCCC12C(=CC(C)CC1CC(=O)CC)C=CC(C)C2CCC1CC(C)CC1C</smiles>

(a)

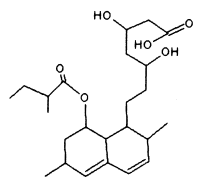

(b) (a) monacolin in lactone form,

(b) monacolin in hydroxy acid form

monacolin $\mathrm{K}(\mathrm{MW}=404)$ which was confirmed by injection of monacolin $\mathrm{K}$ standard.

From the molecular structure of monacolin K, we came to realize that the molecular weight (MW) is 404 . Conclusively, the monacolin $\mathrm{K}$ was found in red yeast from Fujian. Mass spectrum of monacolin $\mathrm{K}$ standard, Mass spectrum of fermented red rice sample preparations as Fig. 2.

Quantitation of Red Yeast Rice and Related Products The content of monacolin $\mathrm{K}$ in red yeast rice and related products were determined by HPLC/PDA and the monacolin $\mathrm{K}$ were calculated with monacolin $\mathrm{K}$ as the reference standard. The results (Table 1) showed that the contents of monacolin $\mathrm{K}$ in these products were considerably different.

Limit of Detection (LOD) and Limit of Quantitation (LOQ) The diluted sample solutions were injected for $10 \mu \mathrm{l}$. The concentration of $0.5 \mu \mathrm{g} / \mathrm{ml}$ resulted in the peak height of monacolin $\mathrm{K}$ approximately three times higher than baseline noise as LOD while $1.5 \mu \mathrm{g} / \mathrm{ml}$ resulted in approximately 10 times higher than baseline noise as LOQ for the ingredient. At the quantitation limit of $1.5 \mu \mathrm{g} / \mathrm{ml}$, the data of six repeated injections indicated repeatability within a deviation of $5-6 \%$ (RSD). 

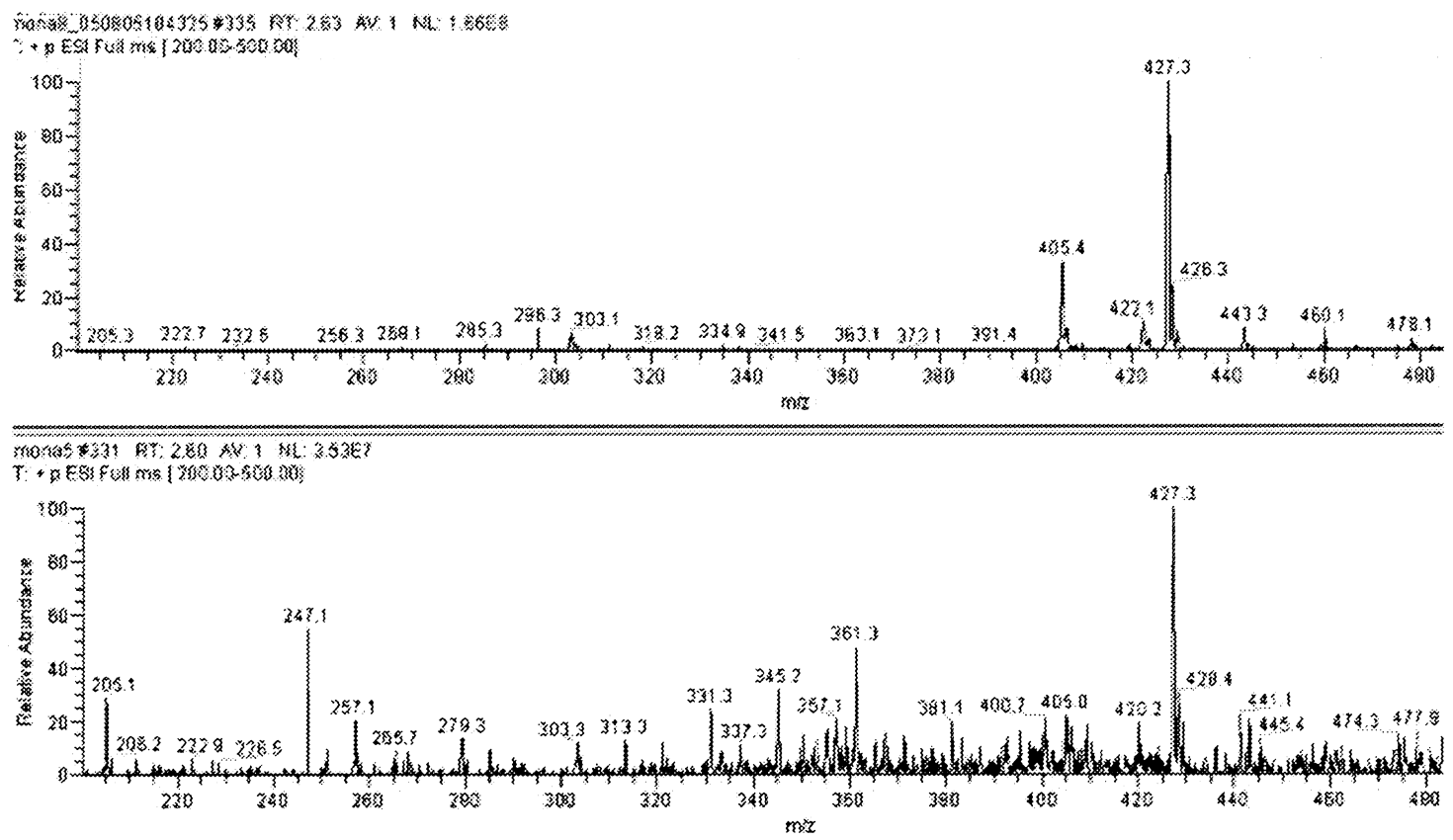

Fig. 2. The Mass Spectrum of Monacolin K Standard and Sample

Table 1. Quantification of Monacolin K in Red Yeast Rice Powder of Different Area

\begin{tabular}{ccccc}
\hline \hline Batch & M+Na & MW & Area & Content (mg/g) \\
\hline P1 & 427 & 404 & Gutiang & 3.1 \\
P2 & 427 & 404 & Jianou & 2.8 \\
P3 & 427 & 404 & Songqi & 2.3 \\
P4 & 427 & 404 & Zhenhe & 2.1 \\
P5 & 427 & 404 & Pinnan & 3.8 \\
P6 & 427 & 404 & Nanping & 3.3 \\
P7 & 427 & 404 & Commercial product & 0.62 \\
P8 & 427 & 404 & Commercial product & 0.31 \\
P9 & 427 & 404 & Commercial product & 0.36 \\
\hline
\end{tabular}

\section{Conclusion}

Monacolin K was characteristic compound produced during fermentation, of which only a part of red yeast rice from Fujian province has been identified in this report. Fujian Pinnan red yeast rice contains plenty of monacolin $\mathrm{K}$. The contents of the monacolin $\mathrm{K}$ were more than the other product. In this respect, further studies are necessary. The fermentation condition might directly effect the production of these secondary metabolites. The combination of LC/PDA and $\mathrm{LC} / \mathrm{PDA} / \mathrm{MS}$ has become a powerful tool to identify and even trace amounts of secondary metabolites like monacolin $\mathrm{K}$ for discriminating forgery or fake of Fujian red yeast rice. Chromatographic chemical profiling proved to be an effective and convenient approach for the qualitative evaluation of this kind of products and could be used for the quality control of the target material as it yielded detailed chemical information involving both the characteristic compound and related constituent. The present method is considered to be applicable to the quantitative and qualitative analysis of monacolin $\mathrm{K}$ in commercial foods and functional foods.

Acknowledgements This research was financially supported by the Key Subject of Fujian Science and Technology Bureau, China.

\section{References}

1) Li Y., Ye Q., Li J., Hua Y., Ju D., Zhang D., Cooper R., Chang M., J. Agric. Food Chem., 48, 5220-5225 (2000).

2) Brosche T., Kral C., Summa J. D., Arch. Gerontol. Geriat., 1996, 207-221 (1996)

3) Wang J. X., Lu Zh. L., Chi J. M., Curr. Ther. Res., 58, 964-978 (1997).

4) Li Ch. L., Zhu Y., Wang Y. Y., Zhu S. Sh., Chang J., Nutr. Res., 18, 71-81 (1998).

5) Heber D., Yip I., Ashley J. M., Elashoff D. A., Am. J. Clin. Nutr., 69, $231-236$ (1999)

6) Journoud M., Jones P. J. H., Life Sci., 74, 2675-2683 (2004).

7) Itoh H., J. Nippon Fermentation Zasshi, 12, 948 -953 (1994).

8) Li Y.-g., Li Y.-g., Fang zhang, Wang Z.-t., Hu Z.-b., J. Pharm. Biomed. Anal., 35, 1101-1112 (2004).

9) Lazarowych N. J., Pekos P., Drug Inform. J., 32, 497-512 (1998).

10) Gong F., Liang Y. Z., Xie P. Sh., J. Chromatogr. A, 1002, 25-40 (2003).

11) Hong X. K., Wang Zh. H., Chin. Trad. Herbal Drugs, 33, 23-27 (2002).

12) Calaf R. E., Carrascal M., Gelpi E., J. Abian, Rapid Commun. Mass Spectrose., 11, 75-80 (1997). 